\title{
On The Dielectric Performance of Modified Epoxy Networks
}

\author{
I. A. Saeedi, A. S. Vaughan and T. Andritsch \\ The Tony Davies High Voltage Laboratory \\ University of Southampton \\ Southampton \\ Southampton, UK, SO17 1BJ \\ iahs1e13@soton.ac.uk
}

\begin{abstract}
Epoxy resins represent a commonly used basis for insulation materials and have been used in many different electrical applications. The formation of these systems involves reactions between a hardener, such as an amine-curing system, and an epoxy terminated resin. Recent studies have reported that epoxy resin systems can exhibit enhanced physical properties when the stoichiometry of the resin is varied using reactive diluent. This has been attributed to the increased free volume within the molecular structures within the epoxy resin network. The work described here set out to investigate this hypothesis concerning the potential benefits of varying the network structure of epoxy resin through the inclusion of monofunctional reactive diluents within the epoxy formulation. This research is of potential significance because any modification of the epoxy resin network results in modified thermal, mechanical and electrical properties and, consequently, represents a potential mean of tailoring overall performance to suit particular applications.
\end{abstract}

Keywords-epoxy resin; reactive diluent; epoxy network; reactive modifier; dielectric properties

\section{INTRODUCTION}

High voltage power generation and transmission applications require insulation materials with excellent dielectric properties. Due to their outstanding electrical and thermal properties, epoxy resins are widely used in high voltage systems. Since the operation efficiency of these systems is directly related to the performance of their epoxy insulations [1], several attempts have been made to vary the properties of these thermosetting materials to suit particular application. For example, the addition of nano- and microsized fillers which improved the thermal properties of epoxy resin in [2] and changing the architecture structure of the epoxy using reactive modifiers which enhanced the mechanical strength of the epoxy matrix used in [3]. As part of a study of modified epoxy resin systems, the network structure was varied using octyl/decyl glycidyl ether [4]. This showed that the addition of epoxy reactive diluent have introduced a high free volume in the system, and changed the molecular dynamics by creating modified network structure with small branches which resulted in varied electrical and thermal properties of the final solid polymer. The study reported here is set out to investigate the effect of adding reactive diluent on the dielectric properties of epoxy resin. The aim of this work is to research the impact of alteration to the epoxy network structure to modify some of the properties of epoxy systems to meet the demands of particular applications. (a) Amine hardener
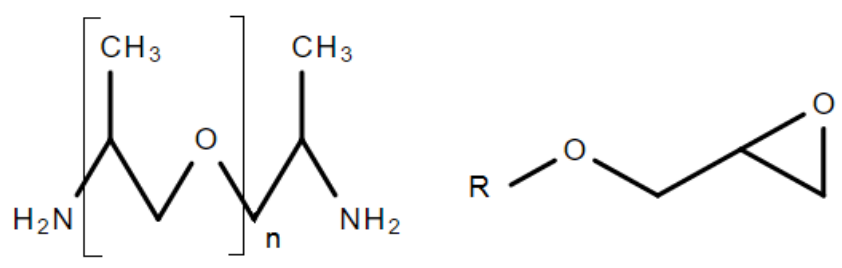

(c) Diglycidyl ether of bisphenol-A<smiles>CC(C)(c1ccc(OCC2CO2)cc1)c1ccc(OCC2CO2)cc1</smiles>

Fig. 1. Chemical Structure of the maerials used in this work.

In this work, we report the impact of introducing varied concentrations of epoxy-based reactive diluent (glycidyl hexadecyl ether) on the thermal and the dielectric properties of epoxy resin (Diglycidyl ether of bisphenol-A). Thus, basically alkyl branches are introduced to the epoxy matrix and their effect on the glass transmission temperature (Tg), the permittivity and the conductivity of epoxy resin will be investigated.

\section{EXPERIMENTAL}

\section{A. Materials}

For this study, samples were produced by mixing three main substances: epoxy resin, mono-functional epoxyterminated reactive diluent and polyether amine hardener shown in Fig. 1. The epoxy resin used was Diglycidyl ether of bisphenol-A (DGEBA) has Epoxide Equivalent Weight EEW of 172-176 g mol-1 (DER332 from Sigma Aldrich). The epoxy resin matrix was modified using 2,3-Epoxypropyl hexadecyl ether reactive diluent (glycidyl hexadecyl ether from Sigma Aldrich). The final system was cured using Jeffamine D-230 hardener with hydrogen equivalent molar mass of $60 \mathrm{~g}$ mol-1 (from Huntsman). Optimum theoretical stoichiometry was used to achieve a complete reaction between all epoxide groups of the epoxy resin and the amine groups of the hardener. This was calculated to be a mass of 344 parts of amine and 1000 part of resin for complete reaction. 


\section{B. Sample Preparation}

The epoxy systems used in this research were prepared by, first weighting out 1000 : 344 part by mass of DER 332 resin : D-230 amine hardener. In addition, a varied percentages $(5 \%$ $15 \%$ parts) of the total mass of reactive diluent were added. Prior to the scaling, the resin and the reactive diluent were preheated in separate beakers for $60 \mathrm{~min}$ at $50{ }^{\circ} \mathrm{C}$ to reduce viscosity. Then, the three components (epoxy, hardener and reactive diluent) were mixed together at room temperature, using magnetic stirrer for $5 \mathrm{~min}$, then the mixture was degassed for another $5 \mathrm{~min}$. After degassing, the mixture was cast in preassembled molds. The resulting samples had a thickness of about $200 \mu \mathrm{m}$. The molds were then placed in a fan oven set at $80^{\circ} \mathrm{C}$ and left to cure for 2 hours before slowly cool down for about 12 hours. The resulting epoxy samples were taken out of the molds and post-cured at $125{ }^{\circ} \mathrm{C}$ for 3 hours. Finally, they were left to cool down to room temperature, before storing them in vacuum until use. Samples used for dielectric spectroscopy test and DC conductivity measurements were gold sputtered to ensure good contact between the epoxy sample and the cell electrode. A uniform thin gold layer was produced on both sides of the sample with deposition current of $25 \mathrm{~mA}$ and rate of $7.5 \mathrm{~nm} / \mathrm{mm}$ with diameter about $30 \mathrm{~mm}$.

\section{Effect of Using Reactive Diluents}

To study the effect of modifying the network structure of epoxy resin, varied percentages of Glycidyl Hexadecyl Ether (GHE) were added to the bi-functional epoxy resin. Throughout this study XGHE notation was used to refer to the samples, where $\mathrm{X}$ corresponds to the percentage of total mass added of GHE diluent. For example, 15 GHE sample contains $15 \%$ added reactive diluent while the designation $0 \mathrm{GHE}$ would correspond to neat epoxy samples.

\section{Material Characterization}

First, the thermal degradation of modified epoxy resin samples was characterized using Thermogravimetric Analysis (TGA) technique. The PerkinElmer TGA 7 Analyzer was set to operate in air at temperature range from $30{ }^{\circ} \mathrm{C}$ to $600{ }^{\circ} \mathrm{C}$ with heating rate of $10{ }^{\circ} \mathrm{C} \mathrm{min}{ }^{-1}$.

Differential Scanning Calorimetry (DSC) method was used to investigate the glass transmission temperature and heat capacity of the study samples. The Perkin Elmer DSC7 was calibrated using pure indium. The glass transmission temperature was recorded for samples with mass of about 10 $\mathrm{mg}$ for two heating scans. Each heating cycle starts from $30^{\circ} \mathrm{C}$ to $170{ }^{\circ} \mathrm{C}$ with heating rate of $10{ }^{\circ} \mathrm{C} \mathrm{min}{ }^{-1}$. Between the two cycles all samples were cooled down to room temperature then rescanned using the same rate used for the previous heating cycle $\left(10{ }^{\circ} \mathrm{C} \mathrm{min}^{-1}\right)$. The data reported here are all from the second heating cycle.

The molecular dynamics of the samples were investigated using Schlumberger SI 1260 impedance / phase gain analyzer which was connected to Solartron 1296 dielectric interface system. The complex permittivity of gold sputtered samples was measured as a function of frequency for the ranges from
$0.1 \mathrm{~Hz}$ to $0.1 \mathrm{MHz}$ at room temperature. The amplitude of the applied AC electric voltage was set to 1 volt.

The DC conductivity of the modified systems was investigated using DC electric field applied from Keithley 6487 picoammeter. The voltage source was connected to 20 $\mathrm{mm}$ diameter electrodes where the test samples were place between the two opposing electrodes. A DC field of $40 \mathrm{kV}$ $\mathrm{mm}^{-1}$ was applied to the gold sputtered samples for 3 hours and measurement were taken at room temperature.

\section{RESULTS}

\section{A. Thermal Analysis}

In order to validate the range of temperature which can be used for the modified systems, the thermal degradation behavior of modified epoxy resin networks was observed using TGA technique. Table I shows the temperatures at which $1 \%$ of the samples weight are lost, which indicates that all samples are very stable with no significant weight change up to around $267{ }^{\circ} \mathrm{C}$. However, at elevated temperatures (above $267^{\circ} \mathrm{C}$ ), the samples undergoes thermal degradation. This insures that the samples are stable and their thermal properties can be studied up to the required temperature of the DSC measurements which is about $170{ }^{\circ} \mathrm{C}$. Fig. 2 along with Table I illustrates the effect of the addition of reactive diluent on the heat capacity and $\mathrm{Tg}$ of epoxy resin samples. As can be seen, Tg of pure samples was observed at about $85.78{ }^{\circ} \mathrm{C}$. Modified epoxy networks have lower Tg than the unmodified systems. As the mass percentage of the added diluent increase, both $\mathrm{Tg}$ and the heat capacity of the modified networks decrease.

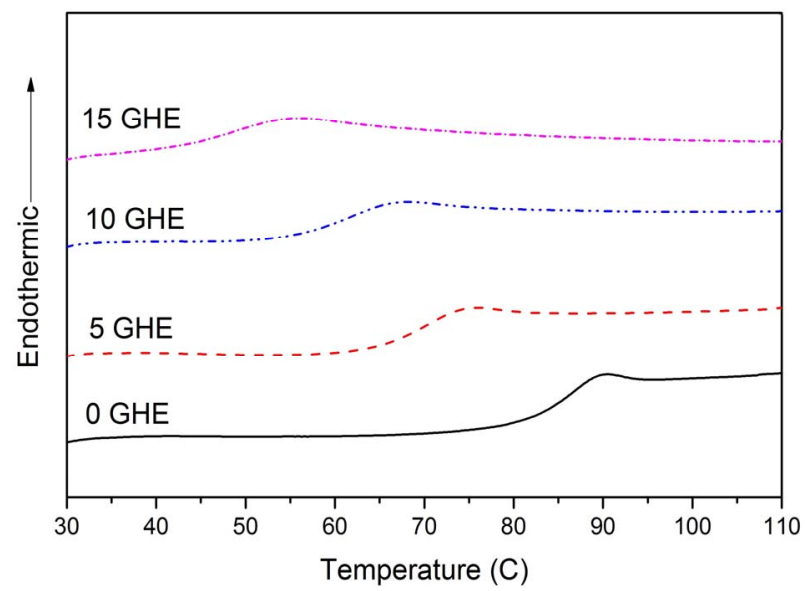

Fig. 2. Differential scanning calorimetry measurments as a function of temperature for neat and modified epoxy systems.

TABLE I. DSC AND TGA DATA OF MODIFIED EPOXY

\begin{tabular}{|c|c|c|c|}
\hline Sample ID & $\boldsymbol{T g}\left({ }^{\circ} \mathbf{C}\right)$ & $\Delta \boldsymbol{C p}\left(\boldsymbol{J} / \boldsymbol{g}^{\circ} \boldsymbol{C}\right)$ & $\begin{array}{c}\boldsymbol{T G A , 1 \%} \text { weight } \\
\text { loss }\left({ }^{\boldsymbol{}} \boldsymbol{C}\right)\end{array}$ \\
\hline $0 \mathrm{GHE}$ & 85.87 & 0.530 & 290 \\
\hline $5 \mathrm{GHE}$ & 68.70 & 0.434 & 294 \\
\hline $10 \mathrm{GHE}$ & 62.20 & 0.274 & 278 \\
\hline $15 \mathrm{GHE}$ & 46.2 & 0.270 & 276 \\
\hline
\end{tabular}




\section{B. Dielectric Spectroscopy}

The dielectric behavior of pure and modified epoxy resin networks was obtained as a function of frequency for real and imaginary part of complex permittivity at room temperature, as shown in Fig. 3a and Fig. 3b, respectively. From Fig. 3a, it can be seen that as the added weight of reactive diluent increase, the real part of complex permittivity decrease. Whilst the imaginary part of complex permittivity (Fig. 3b) shows a slightly increasing peak at low frequency ranges and decreasing peak at frequency below $10^{5} \mathrm{~Hz}$. The variation in the low frequency and high frequency up to $10^{5} \mathrm{~Hz}$ will be discussed in the discussion section. We attribute the large variation in the high frequency measurements (above $10^{5} \mathrm{~Hz}$ ) of the imaginary permittivity to noise limitation of the measurement equipment.

\section{DC Conductivity}

Generally, GHE modified epoxy resin samples have lower DC conductivity than pure epoxy samples, as shown in Fig. 4. The DC conductivity of the GHE modified networks tends to vary with changing the weight of reactive diluent. Sample 15 GHE has the highest DC conductivity which is comparable to the conductivity of the neat epoxy, while sample 10 GHE has the lowest measured DC conductivity. The measured DC current for the samples tends to follow a similar trend to that of the DC conductivity, as illustrated in Table II.
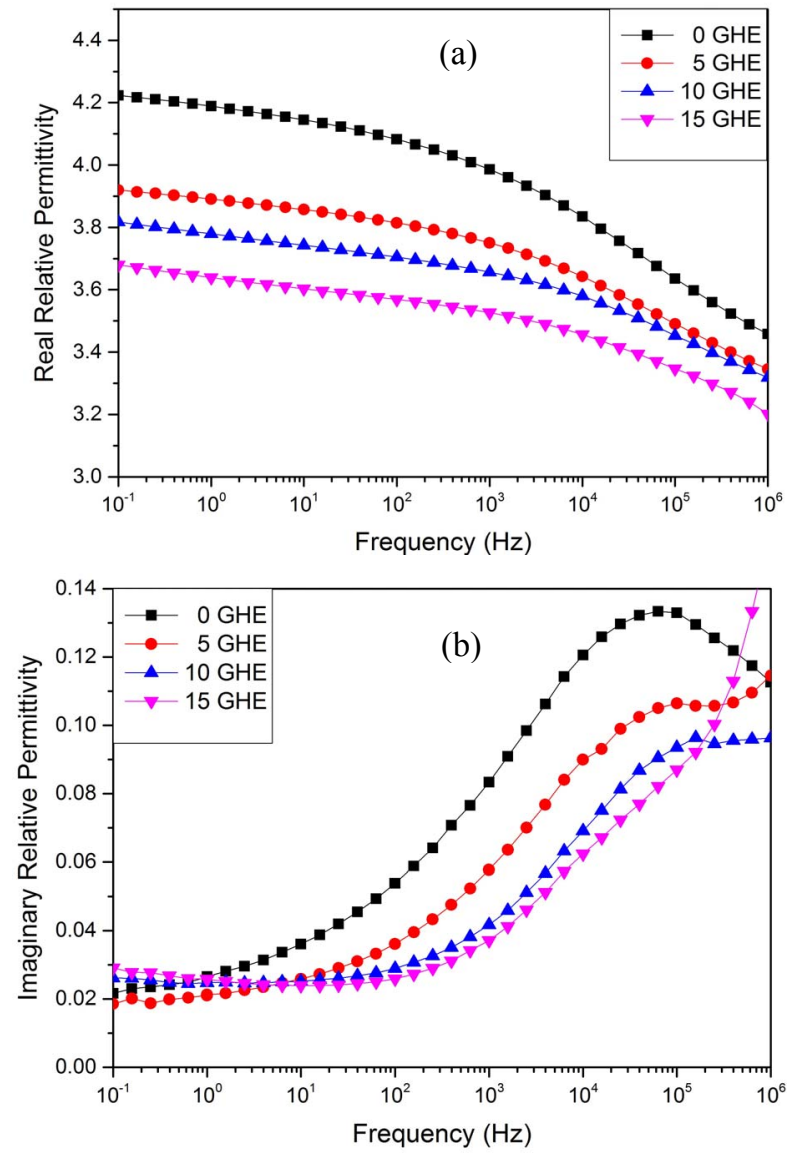

Fig. 3. Dielectric spectroscopy measurements for modified epoxy resin: (a) real part of complex permittivity, (b) imaginary part of complex permittivity

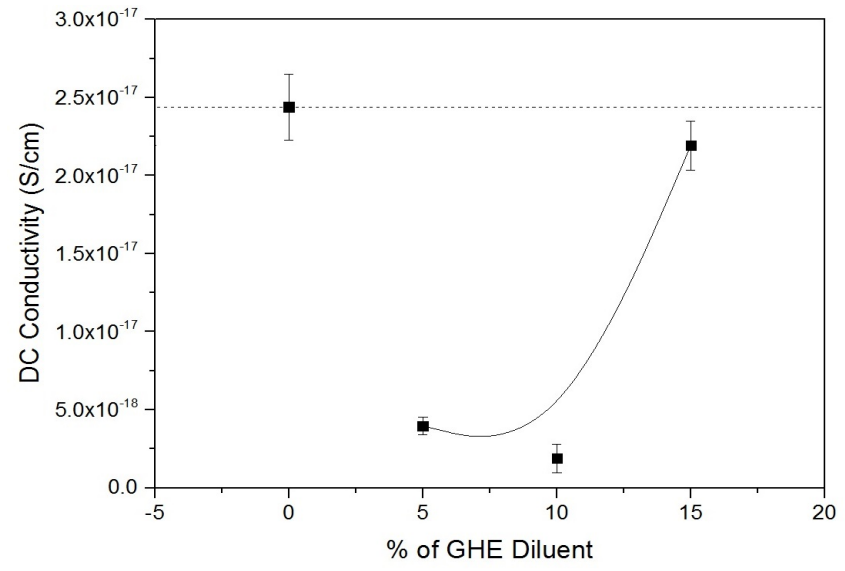

Fig. 4. DC conductivity measurments of neat and modified epoxy systems.

TABLE II. DC CONDUCTIVITY DATA

\begin{tabular}{|c|c|c|c|}
\hline Sample & $\begin{array}{c}\text { Current } \\
(\boldsymbol{A})\end{array}$ & $\begin{array}{c}\text { DC Conductivity } \\
(\text { S/cm })\end{array}$ & Error \\
\hline $0 \mathrm{GHE}$ & $6.9 \times 10^{-11}$ & $2.4388 \times 10^{-17}$ & $2.10491 \times 10^{-18}$ \\
\hline $5 \mathrm{GHE}$ & $1.12 \times 10^{-11}$ & $3.96501 \times 10^{-18}$ & $5.47413 \times 10^{-19}$ \\
\hline $10 \mathrm{GHE}$ & $5.32 \times 10^{-12}$ & $1.89657 \times 10^{-18}$ & $9.16639 \times 10^{-19}$ \\
\hline $15 \mathrm{GHE}$ & $6.21 \times 10^{-11}$ & $2.19194 \times 10^{-17}$ & $1.56839 \times 10^{-18}$ \\
\hline
\end{tabular}

\section{DISCUSSION}

Previous published work [4] on the effect of reactive diluent on the dielectric properties of epoxy resin demonstrated that the change in the epoxy architecture caused decrease in the $\mathrm{Tg}$ as the added weight of reactive modifiers increase. Comparison of this behavior with the data reported here supports the hypothesis that the decrease in Tg can be explained by the fact that the added diluent is a long chain alkyl group $\left(\mathrm{C}_{19} \mathrm{H}_{38} \mathrm{O}_{2}\right)$, which resulted in increased in the free volume in the system and hence caused the noticed decreased Tg.

Studies investigated the effect of $\mathrm{Tg}$ on the dielectric behavior of similar material [5] and modified epoxy networks [4] reported a decrease in the real permittivity of alkyl modified epoxy networks for permittivity test below Tg. Since the current dielectric measurements were conducted at room temperature, which is well below the measured $\mathrm{Tg}$ of the modified epoxy networks, the noticed decrease in the real permittivity is in line with the one described in the previous work [4].

According to Andritsch [6], the variation in the imaginary part of complex permittivity at frequencies below $100 \mathrm{~Hz}$ could be ascribed to $\alpha$-relaxation process at Tg of the network. Since the modified epoxy resin samples of this work showed decrease in the measured $\mathrm{Tg}$, the slight increase in the peak at the low frequencies part of the imaginary permittivity can be attributed to $\alpha$-relaxation affected by the change in the glass transmission temperature of the modified network. This is consistent with dielectric behavior of temperature depend studies of epoxy resin systems reported in [5]. 
The relaxation peak at the high frequency can be attributed to $\beta$-relaxation, which is associated to the mobility of side chains [7]. According to Lui et al. [4], introducing reactive diluent generate an epoxy network with high degree of crosslink than neat epoxy systems due to the introduction of network nodes and small branches. As the epoxy network become highly cross linked the side chains would have smaller ability to movement within the system, which would explain the noticed decrease in $\beta$-relaxation as the parts of added reactive diluent increase.

Finally, Andritsch et al. [8], on a study of the DC conductivity of filled epoxy network, stated that the agglomeration of nanoparticles could have generated regions with easier charge transport than unfilled systems, which resulted in reduced DC conductivity of the filled epoxy samples. Comparing this with the DC conductivity behavior reported in this study, where the increase in the mass of reactive diluent, samples $5 \mathrm{GHE}$ and 15 GHE have increased DC conductivity could be caused by the increase in the free volume which could generated regions with easier charge transport resulted from the introduction of alkyl group to the network (noted by the drop of $\mathrm{Tg}$ ). If this interpretation were correct, then reactive diluents could be performing in a similar fashion to nanoparticles and hence it is possible, therefore, that reactive diluent with suitable R-group may replace the need to use nanoparticles which would avoid the dispersion problem which is one of the major limitation of nanoparticles.

\section{CONCLUSIONS}

The impact of changing the architecture of epoxy resin using epoxy-based reactive diluent have been studied. The introduction of the reactive modifiers changed the free volume in the system, which resulted in significant decrease in $\mathrm{Tg}$, associated with the increased concentrations of added reactive diluent. It was found that the decrease in $\mathrm{Tg}$ caused a steady decline in the permittivity of the modified systems, and could lead to regions with easy charge transport within the system, as a result, a general decrease in the DC conductivity of modified epoxy samples could be observed.

Comparison of the behavior reported in this study with that of epoxy systems filled with nanocomposites suggests, that the added reactive diluents are performing in a similar way to nanoparticles, which was not expected. Therefore, as the two are functioning in the same fashion, it is suggested that reactive diluents might be more suitable to tailor the electrical properties of epoxy resin systems than nanoparticles.

\section{ACKNOWLEDGMENT}

The authors would like to thank the Schlumberger Foundation Faculty for the Future for the financial support.

\section{REFERENCES}

[1] T. Line, "9897 Review Performance Part I : Review [ Iv Transmission Line Insulators in Desert Conditions Research and Methods Adopted Internationally," IEEE Trans. Electr. Insul., no. 2, 1983.

[2] M. Reading, A. S. Vaughan, and P. L. Lewin, "An investigation into improving the breakdown strength and thermal conduction of an epoxy system using boron nitride," in Electrical Insulation and Dielectric Phenomena (CEIDP), IEEE Conference, 2011, pp. 636-639.

[3] L. Núnez-Regueira, M. Villanueva, and I. Fraga-Rivas, "Effect of a reactive diluent on the curing and dynamomechanical properties of an epoxy-diamine system," J. Therm. Anal. Calorim., vol. 86, no. 2, pp. 463-468, 2006

[4] Y. Liu, A. S. Vaughan, I. L. Hosier, C. Yeung, and T. Andritsch, "On epoxy network structure and dielectric performance," in Electrical Insulation and Dielectric Phenomena (CEIDP), IEEE Conference, 2015, pp. 114-117.

[5] C. Yeung, "Spectroscopic Analysis of Nanodielectric Interfaces," Doctoral dissertation, University of Southampton, 2013.

[6] T. Andritsch, "Epoxy Based Nanodielectrics for High Voltage DC Applications: Synthesis, Dielectric Properties and Space Charge Dynamics," Doctoral dissertation, Delft University of Technology, 2010.

[7] R. Böhmer, G. Diezemann, F. Kremer, and A. Schönhals, Broadband Dielectric Spectroscopy. Springer-Verlag Berlin Heidelberg GmbH, 2001.

[8] T. Andritsch, R. Kochetov, P. H. F. Morshuis, and J. J. Smit, "DC conduction in epoxy based nano- and mesocomposites," Electr. Insul. Dielectr. Phenom. (CEIDP), IEEE Conf., 2010. 